\title{
ROUGH MARCINKIEWICZ INTEGRALS RELATED TO SURFACES OF REVOLUTION *
}

\author{
HUSSAIN AL-QASSEM ${ }^{\dagger}$ AND AHMAD AL-SALMAN ${ }^{\dagger}$
}

\begin{abstract}
In this paper, we present a systematic treatment of Marcinkiewicz integrals with block space function kernels and prove the $L^{p}$ boundedness of several classes of Marcinkiewicz integrals along surfaces of revolution. The results in this paper extend as well as improve previously known results.
\end{abstract}

1. Introduction and results. Let $\mathbf{R}^{n}, n \geq 2$ be the $n$-dimensional Euclidean space and $\mathbf{S}^{n-1}$ be the unit sphere in $\mathbf{R}^{n}$ equipped with the normalized Lebesgue measure $d \sigma$.

For a suitable mapping $\Psi: \mathbf{R}^{n} \rightarrow \mathbf{R}^{m}$, we define the Marcinkiewicz integral operator $\mathcal{M}_{\Psi, \Omega, h}$ by

$$
\mathcal{M}_{\Psi, \Omega, h} f(x)=\left(\int_{\mathbf{R}}\left|\zeta_{t, \Psi} f(x)\right|^{2} d t\right)^{\frac{1}{2}},
$$

where

$$
\zeta_{t, \Psi} f(x)=\frac{1}{2^{t}} \int_{|y| \leq 2^{t}} f(x-\Psi(y)) h(|y|) \frac{\Omega(y)}{|y|^{n-1}} d y,
$$

$h(\cdot)$ is a measurable function on $\mathbf{R}^{+}$, and $\Omega$ is a homogeneous function of degree 0 , integrable over $\mathbf{S}^{n-1}$, and satisfies

$$
\int_{\mathbf{S}^{n-1}} \Omega(x) d \sigma(x)=0 .
$$
by $\mathcal{M}_{\Omega}$.

If $h=1, m=n$, and $\Psi(y)=\left(y_{1}, \ldots, y_{n}\right)$ we shall denote the operator $\mathcal{M}_{\Psi, \Omega, h}$

E. M. Stein introduced the operator $\mathcal{M}_{\Omega}$ and showed that if $\Omega \in \operatorname{Lip}_{\alpha}\left(\mathbf{S}^{n-1}\right)$, $(0<\alpha \leq 1)$, then $\mathcal{M}_{\Omega}$ is of type $(p, p)$ for $p \in(1,2]$ and of weak type $(1,1)$ (see [St1]). Subsequently, A. Benedek, A. Calderón, and R. Panzone proved that $\mathcal{M}_{\Omega}$ is of type $(p, p)$ for $p \in(1, \infty)$ if $\Omega \in C^{1}\left(\mathbf{S}^{n-1}\right)$ (see [BCP]).Very recently, the study of the more general class of operators $\mathcal{M}_{\Psi, \Omega, h}$ for various mappings $\Psi$ and under various conditions on $\Omega$ has attracted the attention of many authors (see, for example, [AsAq], [CFP], [DFP], [DP]).

On the other hand, there has been a considerable amount of research concerning the $L^{p}$ boundedness of singular integrals along surfaces of revolution. For relevant results one may consult $[\mathrm{AqP}],[\mathrm{AsP}],[\mathrm{CF}],[\mathrm{KWWZ}]$, [LPY], among others.

In this paper, we shall investigate the $L^{p}$ boundedness of Marcinkiewicz integrals along surfaces of revolution $\Psi=\Psi_{\phi}=\left\{(y, \phi(|y|)): y \in \mathbf{R}^{n}\right\}$ for various mappings $\phi$ and when $\Omega \in B_{q}^{0,0}\left(\mathbf{S}^{n-1}\right), q>1$, where $B_{q}^{0,0}\left(\mathbf{S}^{n-1}\right)$ represents a special class of block spaces which will be recalled in Section 3. Here our Marcinkiewicz integral operator

\footnotetext{
*Received December 5, 2001; accepted for publication May 27, 2003.

†Department of Mathematics, Yarmouk University, Irbid-Jordan (husseink@yu.edu.jo; alsalman@yu.edu.jo).
} 
$\mathcal{M}_{\Psi_{\phi}, \Omega, h}$ will be denoted by $\mathcal{M}_{\phi, \Omega, h}$. It should be remarked that $B_{q}^{0,0}\left(\mathbf{S}^{n-1}\right)$ contains $L^{q}\left(\mathbf{S}^{n-1}\right)$ as a proper subspace for each $q>1$ and

$$
\bigcup_{q>1} L^{q}\left(\mathbf{S}^{n-1}\right) \stackrel{\subsetneq}{\neq} \bigcup_{q>1} B_{q}^{0,0}\left(\mathbf{S}^{n-1}\right) .
$$
if

For a measurable real valued function $h$ on $\mathbf{R}^{+}$, we say that $h \in \Delta_{\gamma}\left(\mathbf{R}^{+}\right), \gamma>1$,

$$
\|h\|_{\Delta_{\gamma}}=\sup _{R>0}\left\{R^{-1} \int_{R}^{2 R}|h(t)|^{\gamma} d t\right\}^{\frac{1}{\gamma}}<\infty .
$$

Our main results are the following:

Theorem 1.1. Assume that $\Omega \in B_{q}^{0,0}\left(\mathbf{S}^{n-1}\right), h \in \Delta_{\gamma}\left(\mathbf{R}^{+}\right)$for some $q, \gamma>1$ and $\phi$ is in $C^{2}([0, \infty))$, convex, and increasing. Then for every $p$ satisfying $|1 / p-1 / 2|<\min \left\{1 / 2,1 / \gamma^{\prime}\right\}$, there exists a constant $C_{p}$ such that

$$
\left\|\mathcal{M}_{\phi, \Omega, h}(f)\right\|_{L^{p}\left(\mathbf{R}^{n+1}\right)} \leq C_{p}\|f\|_{L^{p}\left(\mathbf{R}^{n+1}\right)}
$$

for every $f \in L^{p}\left(\mathbf{R}^{n+1}\right)$.

THEOREM 1.2. Suppose that $\Omega \in B_{q}^{0,0}\left(\mathbf{S}^{n-1}\right)$, and $h \in \Delta_{\gamma}\left(\mathbf{R}^{+}\right)$for some $q, \gamma>1$. Suppose that $\phi$ is a continuous function on $[0, \infty)$ and $\phi \in C^{1}((0, \infty))$ such that (i) $\phi$ is strictly increasing function on $[0, \infty)$; (ii) $\phi^{\prime}(t) \geq C \frac{\phi(t)}{t}$ for $t>0$ and $C>0$, and (iii) $\phi(2 t) \leq c \phi(t)$ for $t>0$ and $c>0$. Then (1.3) holds for every $f \in L^{p}\left(\mathbf{R}^{n+1}\right)$.

THEOREM 1.3. Assume that $\Omega \in B_{q}^{0,0}\left(\mathbf{S}^{n-1}\right), h \in \Delta_{\gamma}\left(\mathbf{R}^{+}\right)$for some $q, \gamma>1$ and $\phi:(0, \infty) \rightarrow \mathbf{R}$ is a smooth function which satisfies the following growth conditions: (i) $|\phi(t)| \leq C_{1} t^{d}$, (ii) $C_{2} t^{d-1} \leq\left|\phi^{\prime}(t)\right| \leq C_{3} t^{d-1}$, (iii) $\left|\phi^{\prime \prime}(t)\right| \leq C_{4} t^{d-2}$, for some $d \neq 0$ and $t \in(0, \infty)$, where $C_{1}, C_{2}, C_{3}$, and $C_{4}$ are positive constants independent of $t$. Then (1.3) holds for every $f \in L^{p}\left(\mathbf{R}^{n+1}\right)$.

THEOREM 1.4. Assume that $\Omega \in B_{q}^{0,0}\left(\mathbf{S}^{n-1}\right), h \in \Delta_{\gamma}\left(\mathbf{R}^{+}\right)$for some $q, \gamma>1$ and $\phi$ is a polynomial. Then (1.3) holds for every $f \in L^{p}\left(\mathbf{R}^{n+1}\right)$.

It is worth pointing out that using the same argument as in [DFP] we are only able to obtain Theorems 1.1-1.3 under the stronger condition $\Omega \in L^{q}\left(\mathbf{S}^{n-1}\right), q>1$. Therefore, it is imperative to attack the problems under considerations through a proper decomposition of our operators along with keeping track of certain constants. In fact, the proof of our results will be a consequence of two general theorems stated in Section 2. We shall present a systematic method which not only allows us to obtain the $L^{p}$ boundedness of Macinkiewicz integral operators under considerations, but also has shown to be useful in handling some other problems in this area which will appear in forth coming papers. 
2. General results. Given a family of measures $\left\{\sigma_{t}: t \in \mathbf{R}\right\}$, we define the maximal operator $\sigma^{*}$ by $\sigma^{*}(f)=\sup _{t \in \mathbf{R}}|| \sigma_{t}|* f|$. Also, we write $t^{ \pm \alpha}=\inf \left\{t^{\alpha}, t^{-\alpha}\right\}$ and $|\sigma|$ for the total variation of $\sigma$, which is a positive measure.

LEMMA 2.1. Let $\left\{\sigma_{t}: t \in \mathbf{R}\right\}$ be a family of Borel measures on $\mathbf{R}^{n}$ such that $\left\|\sigma_{t}\right\| \leq 1$. Assume that

$$
\left\|\sigma^{*}(f)\right\|_{q} \leq B\|f\|_{q} \text { for some } q>1 \text { and } B>1 .
$$

Then the inequality

$$
\left\|\left(\int_{-\infty}^{\infty}\left|\sigma_{t} * g_{t}\right|^{2} d t\right)^{\frac{1}{2}}\right\|_{p_{0}} \leq \sqrt{B}\left\|\left(\int_{-\infty}^{\infty}\left|g_{t}\right|^{2} d t\right)^{\frac{1}{2}}\right\|_{p_{0}}
$$

holds for $\left|1 / p_{0}-1 / 2\right|=1 /(2 q)$ and for arbitrary measurable functions $g(t, x)=g_{t}(x)$ defined on $\mathbf{R} \times \mathbf{R}^{n}$.

Proof. We use a similar argument as in [Du]. Since $\left\|\sigma_{t}\right\| \leq 1$ we immediately get

$$
\left\|\int_{-\infty}^{\infty}\left|\sigma_{t} * g_{t}\right| d t\right\|_{1} \leq\left\|\int_{-\infty}^{\infty}\left|g_{t}\right| d t\right\|_{1}
$$

On the other hand,

$$
\left\|\sup _{t \in \mathbf{R}}\left|\sigma_{t} * g_{t}\right|\right\|_{q} \leq\left\|\sigma^{*}\left(\sup _{t \in \mathbf{R}}\left|g_{t}\right|\right)\right\|_{q} \leq B\left\|\sup _{t \in \mathbf{R}}\left(\left|g_{t}\right|\right)\right\|_{q} .
$$

By interpolation between (2.3) and (2.4) we get (2.2) when $1 / p_{0}=(1 / 2)(1+1 / q)$. The case $p_{0}>2$ follows by duality.

Theorem 2.2. Let $L: \mathbf{R}^{n} \rightarrow \mathbf{R}^{m}$ be a linear transformation and $\left\{\sigma_{t}: t \in \mathbf{R}\right\}$ be a family of Borel measures on $\mathbf{R}^{n}$ such that

(i) $\left\|\sigma_{t}\right\| \leq 1$

(ii) $\left|\hat{\sigma}_{t}(\xi)\right| \leq\left(a^{t}|L(\xi)|\right)^{ \pm \frac{\alpha}{B}}$

for some constants $a \geq 2$ and $B>1$. Assume that for some $p_{0}>2$ and for arbitrary functions $g_{t}(x)$ defined on $\mathbf{R} \times \mathbf{R}^{n}$, we have

$$
(i i i)\left\|\left(\int_{-\infty}^{\infty}\left|\sigma_{t} * g_{t}\right|^{2} d t\right)^{\frac{1}{2}}\right\|_{p_{0}} \leq \sqrt{B}\left\|\left(\int_{-\infty}^{\infty}\left|g_{t}\right|^{2} d t\right)^{\frac{1}{2}}\right\|_{p_{0}} .
$$

Then for $p_{0}^{\prime}<p<p_{0}$, there exists a positive constant $C_{p}$ such that

$$
\left\|\left(\int_{-\infty}^{\infty}\left|\sigma_{t} * f\right|^{2} d t\right)^{\frac{1}{2}}\right\|_{p} \leq C_{p} B\|f\|_{p}
$$

for all $f \in L^{p}\left(\mathbf{R}^{n}\right)$. The constant $C_{p}$ is independent of $B$ and the linear transformation $L$.

Proof. Clearly, we may assume that $0<\alpha \leq 1$. By the arguments in the proof of Lemma 6.2 in [FP], we may assume without loss of generality that $m \leq n$ 
and $L=\pi_{m}^{n}$. By an elementary procedure choose a collection of smooth functions $\left\{\Phi_{t, B}\right\}_{t \in \mathbf{R}}$ on $(0, \infty)$ with the following properties: for each $t \in \mathbf{R}$,

$$
\begin{gathered}
0 \leq \Phi_{t, B} \leq 1, \quad \sum_{k \in \mathbf{Z}} \Phi_{k+t, B}(u)=1, \\
\operatorname{supp} \Phi_{t, B} \subseteq\left\{u: a^{-(t+1) B}<u<a^{-(t-1) B}\right\},\left|\frac{d^{s} \Phi_{t, B}(u)}{d u^{s}}\right| \leq \frac{C}{u^{s}}
\end{gathered}
$$

where $C$ can be chosen to be independent of the constant $B$.

Let $T(f)=\left(\int_{-\infty}^{\infty}\left|\sigma_{t} * f\right|^{2} d t\right)^{\frac{1}{2}}$ and $T_{k, B}(f)=\left(\int_{-\infty}^{\infty}\left|\sigma_{B t} * \Psi_{k+t, B} * f\right|^{2} d t\right)^{\frac{1}{2}}$ where $\widehat{\Psi_{t, B}}(\xi)=\Phi_{t, B}\left(\left|\pi_{m}^{n} \xi\right|\right)$. Then it is easy to see that the following inequality

$$
T f(x) \leq \sqrt{B} \sum_{k \in \mathbf{Z}} T_{k, B} f(x)
$$

holds for $f \in \mathcal{S}\left(\mathbf{R}^{n}\right)$. Therefore, to prove (2.6), it suffices to prove

$$
\left\|T_{k, B}(f)\right\|_{p} \leq C_{p} \sqrt{B} 2^{-\alpha \beta_{p}(|k|-1)}\|f\|_{p}
$$

for some positive constants $\beta_{p}$ and $C_{p}$ and for all $p_{0}^{\prime}<p<p_{0}$.

The proof of (2.7) follows by interpolation between a sharp $L^{2}$ estimate and a cruder $L^{p_{0}}$ estimate.

First,

$$
\begin{aligned}
\left\|T_{k, B}(f)\right\|_{p_{0}} & =\frac{1}{\sqrt{B}}\left\|\left(\int_{-\infty}^{\infty}\left|\sigma_{t} * \Psi_{k+\frac{t}{B}, B} * f\right|^{2} d t\right)^{\frac{1}{2}}\right\|_{p_{0}} \\
& \leq\left\|\left(\int_{-\infty}^{\infty}\left|\Psi_{k+\frac{t}{B}, B} * f\right|^{2} d t\right)^{\frac{1}{2}}\right\|_{p_{0}} \\
& \leq C_{p_{0}} \sqrt{B}\|f\|_{p_{0}} .
\end{aligned}
$$

The first inequality follows by (2.5) and the second inequality follows by a trivial change of variable and the same argument as in the proof of (20) in [St2], page 27.

On the other hand, the $L^{2}$ boundedness of $T_{k, B}$ is provided by a simple application of Plancherel's theorem. If $k \geq 0$,

$$
\begin{aligned}
\left\|T_{k, B}(f)\right\|_{2}^{2} & =\int_{-\infty}^{\infty} \int_{\mathbf{R}^{n}}\left|\Phi_{k+t, B}\left(\left|\pi_{m}^{n} \xi\right|\right)\right|^{2}\left|\hat{\sigma}_{B t}(\xi)\right|^{2}|\hat{f}(\xi)|^{2} d \xi d t \\
& \leq \int_{\mathbf{R}^{n}}|\hat{f}(\xi)|^{2}\left(\int_{\left.\left.\left(\log a^{A}\right)^{-1} \log \left(a^{-(k+1) B} \mid \pi_{m}^{n} \xi\right)\right|^{-1}\right)}^{\left(\log a^{A}\right)^{-1} \log \left(a^{-(k-1) B}\left|\pi_{m}^{n} \xi\right|^{-1}\right)}\left(a^{t B}\left|\pi_{m}^{n} \xi\right|\right)^{\frac{2 \alpha}{B}} d t\right) d \xi \\
& \leq 2 a^{-2 \alpha(k-1)}\|f\|_{2}^{2} .
\end{aligned}
$$

Similarly, if $k<0$, we get

$$
\left\|T_{k, B}(f)\right\|_{2} \leq \sqrt{2} a^{\alpha} a^{\alpha k}\|f\|_{2} .
$$

By combining (2.9) and (2.10), we obtain

$$
\left\|T_{k, B}(f)\right\|_{2} \leq \sqrt{2} a^{\alpha} a^{-\alpha|k|}\|f\|_{2} .
$$


By (2.8), (2.11) and applying the Riesz-Thorin Interpolation Theorem for sublinear operators we get (2.7). This finishes the proof of our theorem.

Let us now establish the following theorem on maximal functions.

For given two families $\left\{\mu_{t}: t \in \mathbf{R}\right\}$ and $\left\{\tau_{t}: t \in \mathbf{R}\right\}$ of non negative Borel measures on $\mathbf{R}^{n}$ we define the corresponding maximal functions $\mu^{*}$ and $\tau^{*}$ by

$$
\mu^{*}(f)=\sup _{t \in \mathbf{R}}\left|\mu_{t} * f\right| \text { and } \tau^{*}(f)=\sup _{t \in \mathbf{R}}\left|\tau_{t} * f\right| .
$$

We have the following theorem.

THEOREM 2.3. Let $\left\{\mu_{t}\right\}_{t \in \mathbf{R}}$ and $\left\{\tau_{t}\right\}_{t \in \mathbf{R}}$ be families of non negative Borel measures on $\mathbf{R}^{n}$. Let $L: \mathbf{R}^{n} \rightarrow \mathbf{R}^{m}$ be a linear transformation. Suppose that for all $t \in \mathbf{R}, \xi \in \mathbf{R}^{n}$, for some $a \geq 2, \alpha, C>0$ and for some constant $B>1$ we have

(i) $\left\|\mu_{t}\right\| \leq 1 ;\left\|\tau_{t}\right\| \leq 1$;

(ii) $\left|\hat{\mu}_{t}(\xi)\right| \leq C\left(a^{t}|L(\xi)|\right)^{-\frac{\alpha}{B}}$;

(iii) $\left|\hat{\mu}_{t}(\xi)-\hat{\tau}_{t}(\xi)\right| \leq C\left(a^{t}|L(\xi)|\right)^{\frac{\alpha}{B}}$;

(iv) For any nonnegative function $f, x \in \mathbf{R}^{n}$, the function $h_{x}(t)=a^{t}\left|\mu_{t} * f(x)\right|$ is an increasing function in $t$;

(v) For all $1<p \leq \infty$ and $f \in L^{p}\left(\mathbf{R}^{n}\right)$,

$$
\left\|\tau^{*}(f)\right\|_{p} \leq B\|f\|_{p}
$$

Then the inequality

$$
\left\|\mu^{*}(f)\right\|_{p} \leq B\|f\|_{p}
$$

holds for all $1<p \leq \infty$ and $f$ in $L^{p}\left(\mathbf{R}^{n}\right)$. The constant $C_{p}$ is independent of $B$ and the linear transformation $L$.

Proof. Without loss of generality we may assume that $m \leq n, L=\pi_{m}^{n}$ and $0<\alpha \leq 1$. Let $\psi \in \mathcal{S}\left(\mathbf{R}^{m}\right)$ be a Schwartz function such that $\hat{\psi}(x)=1$ for $|x| \leq 1 / 2$ and $\hat{\psi}(x)=0$ for $|x| \geq 1$. Define the family of measures $\left\{\lambda_{t}: t \in \mathbf{R}\right\}$ by

$$
\hat{\lambda}_{t}(\xi)=\hat{\mu}_{t}(\xi)-\hat{\psi}\left(a^{t} \pi_{m}^{n} \xi\right) \hat{\tau}_{t}(\xi)
$$

By (i)-(iii) and (2.14) we get

$$
\left|\hat{\lambda}_{t}(\xi)\right| \leq C\left(a^{t}\left|\pi_{m}^{n} \xi\right|\right)^{ \pm \frac{\alpha}{B}} \quad \text { for } \xi \in \mathbf{R}^{n} .
$$

Let

$$
g_{\lambda}(f)(x)=\left(\int_{-\infty}^{\infty}\left|\lambda_{t} * f(x)\right|^{2} d t\right)^{\frac{1}{2}} \text { and } \lambda^{*}(f)=\sup _{t \in \mathbf{R}}|| \lambda_{t}|* f|
$$

Then by condition (iv) and (2.14) we have

$$
\mu^{*}(f)(x) \leq g_{\lambda}(f)(x)+C\left(M_{\mathbf{R}^{m}} \otimes i d_{\mathbf{R}^{n-m}}\right)\left(\tau^{*}(f)(x)\right),
$$




$$
\lambda^{*}(f)(x) \leq g_{\lambda}(f)(x)+2 C\left(M_{\mathbf{R}^{m}} \otimes i d_{\mathbf{R}^{n-m}}\right)\left(\tau^{*}(f)(x)\right)
$$

where $M_{\mathbf{R}^{m}}$ is the classical Hardy-Littlewood maximal function on $\mathbf{R}^{m}$ which is bounded in $L^{p}\left(\mathbf{R}^{m}\right)$ for $1<p \leq \infty$. By (2.15) and Plancherel's theorem we obtain

$$
\left\|g_{\lambda}(f)\right\|_{2} \leq C \sqrt{B}\|f\|_{2}
$$

which when combined with (2.12) and (2.17) gives that

$$
\left\|\lambda^{*}(f)\right\|_{2} \leq C B\|f\|_{2}
$$

with $C$ independent of $B$. Thus, by applying Lemma 2.1 with $p_{0}=4$ and $q=2$, we have

$$
\left\|\left(\int_{-\infty}^{\infty}\left|\sigma_{t} * g_{t}\right|^{2} d t\right)^{\frac{1}{2}}\right\|_{p_{0}} \leq C \sqrt{B}\left\|\left(\int_{-\infty}^{\infty}\left|g_{t}\right|^{2} d t\right)^{\frac{1}{2}}\right\|_{p_{0}}
$$

for arbitrary functions $g_{t}(x)$ defined on $\mathbf{R} \times \mathbf{R}^{n}$. By taking $p_{0}=4$ and invoking Theorem 2.2, we get that

$$
\left\|g_{\lambda}(f)\right\|_{p} \leq C_{p} B\|f\|_{p}
$$

for $4 / 3<p<4$ and $f \in L^{p}\left(\mathbf{R}^{n}\right)$ with a positive constant $C_{p}$ independent of $B$. A new application of Theorem 2.2 gives

$$
\left\|g_{\lambda}(f)\right\|_{p} \leq C_{p} B\|f\|_{p}
$$

for $8 / 7<p<8$. By repeating this process, we obtain that

$$
\left\|g_{\lambda}(f)\right\|_{p} \leq C_{p} B\|f\|_{p}
$$

for $1<p<\infty$ and $f \in L^{p}\left(\mathbf{R}^{n}\right)$. Therefore, by (2.12), (2.16) and (2.23) we obtain (2.13) for $1<p<\infty$ and $f \in L^{p}\left(\mathbf{R}^{n}\right)$. The proof of our theorem in now complete.

3. Definitions and some basic lemmas. Let us begin by recalling the definition of a block function on $\mathbf{S}^{n-1}$.

Definition 3.1. For $1<q \leq \infty$ we say that a measurable function $b(\cdot)$ on $\mathbf{S}^{n-1}$ is a $q$-block if it satisfies the following: (i) supp $(b) \subseteq I$ and $(i i)\|b\|_{L^{q}} \leq|I|^{-\frac{1}{q^{t}}}$, where $I$ is an interval on $\mathbf{S}^{n-1}$; i.e.,

$$
I=\left\{x^{\prime} \in \mathbf{S}^{n-1}:\left|x^{\prime}-x_{0}^{\prime}\right|<\alpha \text { for some } \alpha>0, x_{0}^{\prime} \in \mathbf{S}^{n-1}\right\} \text { and }|I|=\sigma(I) .
$$

The block spaces $B_{q}^{0,0}$ on $\mathbf{S}^{n-1}$ are defined as follows:

Definition 3.2. The function space $B_{q}^{0,0}\left(\mathbf{S}^{n-1}\right), 1<q \leq \infty$, consists of all functions $\Omega \in L^{1}\left(\mathbf{S}^{n-1}\right)$ of the form $\Omega=\sum_{\mu=1}^{\infty} c_{\mu} b_{\mu}$ where $c_{\mu} \in \mathbf{C}$; each $b_{\mu}$ is a $q$-block supported in an interval $I_{\mu}$; and

$$
M_{q}^{0,0}\left(\left\{c_{\mu}\right\}\right)=\sum_{\mu=1}^{\infty}\left|c_{\mu}\right|\left(1+\log \left|I_{\mu}\right|^{-1}\right)<\infty
$$


The special class of functions $B_{q}^{0,0}$ were first introduced by Jiang and Lu in their study of singular integral operators of Calderón-Zygmund type (see [LTW]).

For suitable mappings $\phi:[0, \infty) \rightarrow \mathbf{R}, h: \mathbf{R}^{+} \rightarrow \mathbf{R}$ and $\tilde{b}: \mathbf{S}^{n-1} \rightarrow \mathbf{R}$, we define the family of measures $\left\{\sigma_{t, \tilde{b}, h}: t \in \mathbf{R}\right\}$ and the related maximal operator $\sigma_{\tilde{b}, h}^{*}$ on $\mathbf{R}^{n+1}$ by

$$
\begin{aligned}
\int_{\mathbf{R}^{n+1}} f d \sigma_{t, \tilde{b}, h} & =2^{-t} \int_{|y|<2^{t}} f(y, \phi(|y|)) h(|y|) \frac{\tilde{b}\left(y^{\prime}\right)}{|y|^{n-1}} d y, \\
\sigma_{\tilde{b}, h}^{*}(f) & =\sup _{t \in \mathbf{R}}|| \sigma_{t, \tilde{b}, h}|* f| .
\end{aligned}
$$

LEMMA 3.3. Let $h \in \Delta_{\gamma}\left(\mathbf{R}^{+}\right)$for some $\gamma, 1<\gamma \leq 2, \phi(\cdot)$ is in $C^{1}$ of $(0, \infty)$ and $\tilde{b}$ be a function on $\mathbf{S}^{n-1}$ satisfying the following conditions: (i) $\|\tilde{b}\|_{q} \leq|I|^{-\frac{1}{q^{\prime}}}$ for some $q>1$ and for some interval I on $\mathbf{S}^{n-1}$; (ii) $\int_{\mathbf{S}^{n-1}} \tilde{b}(u) d \sigma(u)=0 ;$ (iii) $\|\tilde{b}\|_{1} \leq$

1. Then there exist constants $C$ and $0<\beta<1 / q^{\prime}$ such that

$$
\begin{array}{ll}
\left|\hat{\sigma}_{t, \tilde{b}, h}(\xi, \tau)\right| \leq C\|h\|_{\Delta_{\gamma}}\left|2^{t} \xi\right|^{ \pm \frac{\beta}{\gamma^{\prime} \log |I|}} & \text { if }|I|<e^{-1} \text { and } \\
\left|\hat{\sigma}_{t, \tilde{b}, h}(\xi, \tau)\right| \leq C\|h\|_{\Delta_{\gamma}}\left|2^{t} \xi\right|^{ \pm \frac{\beta}{\gamma^{\prime}}} & \text { if }|I| \geq e^{-1}
\end{array}
$$

for all $t \in \mathbf{R}, \xi \in \mathbf{R}^{n}$, and $\tau \in \mathbf{R}$. The constant $C$ is independent of $t, \tilde{b}, \xi, \tau$ and $\phi(\cdot)$.

Proof. We shall only prove (3.4) and the proof of (3.5) will be easier. By Hölder's inequality and noticing that $\left|I_{t}(s, \xi)\right| \leq 1$ we have

$$
\left|\hat{\sigma}_{t, \tilde{b}, h}(\xi, \tau)\right| \leq\|h\|_{\Delta_{\gamma}}\left(\int_{0}^{1}\left|I_{t}(s, \xi)\right|^{2} d t\right)^{\frac{1}{\gamma^{\prime}}}
$$

where

$$
I_{t}(s, \xi)=\int_{\mathbf{S}^{n-1}} e^{i\left(2^{t} s \xi \cdot x+\tau \phi\left(2^{t} s\right)\right)} \tilde{b}(x) d \sigma(x)
$$

However,

$$
\begin{aligned}
\left|\int_{0}^{1} e^{i 2^{t} s \xi \cdot(x-y)} d s\right| & \leq C \min \left\{1,\left|2^{t} \xi \cdot(x-y)\right|^{-1}\right\} \\
& \leq C\left|2^{t} \xi\right|^{-\beta}\left|\xi^{\prime} \cdot(x-y)\right|^{-\beta}
\end{aligned}
$$

and

$$
\left|I_{t}(s, \xi)\right|^{2}=\int_{\mathbf{S}^{n-1} \times \mathbf{S}^{n-1}} \tilde{b}(x) \overline{\tilde{b}(y)} e^{i 2^{t} s(x-y) \cdot \xi} d \sigma(x) d \sigma(y)
$$

where $\xi^{\prime}=\xi /|\xi|$, and $\beta>0$ with $0<\beta q^{\prime}<1$. Therefore, by Hölder's inequality we get

$$
\left|\hat{\sigma}_{t, \tilde{b}, h}(\xi, \tau)\right| \leq C\|h\|_{\Delta_{\gamma}}\left|2^{t} \xi\right|^{-\frac{\beta}{\gamma^{\prime}}}\|\tilde{b}\|_{q}^{\frac{2}{\gamma^{\prime}}}\left\{\int_{\mathbf{S}^{n-1} \times \mathbf{S}^{n-1}}\left|x_{1}-y_{1}\right|^{-\beta q^{\prime}} d \sigma(x) d \sigma(y)\right\}^{\frac{1}{\gamma q^{\prime}}}
$$


By using (i) and noticing that the last integral is finite, one obtains that

$$
\left|\hat{\sigma}_{t, \tilde{b}, h}(\xi, \tau)\right| \leq C\|h\|_{\Delta_{\gamma}}|I|^{-\frac{2}{q^{\prime} \gamma^{\prime}}}\left|2^{t} \xi\right|^{-\frac{\beta}{\gamma^{\prime}}} .
$$

By combining the preceding estimate with the trivial estimate $\left|\hat{\sigma}_{t, \tilde{b}, h}(\xi, \tau)\right| \leq\|h\|_{\Delta_{\gamma}}$ we obtain (3.4) with a plus sign in the exponent.

On the other hand, by the conditions (ii)-(iii) on $\tilde{b}$ we obtain

$$
\left|\hat{\sigma}_{t, \tilde{b}, h}(\xi, \tau)\right| \leq\|h\|_{\Delta_{\gamma}}\left|2^{t} \xi\right|
$$

which, when combined with the trivial estimate $\left|\hat{\sigma}_{t, \tilde{b}, h}(\xi, \tau)\right| \leq\|h\|_{\Delta_{\gamma}}$, yields the second estimate in (3.4). This finishes the proof of our lemma.

LEMMA 3.4. Let $\phi$ be a function given as in any one of the Theorems 1.1-1.3. Define the maximal function $\mathcal{M}_{\phi}$ by

$$
\mathcal{M}_{\phi} f(u)=\sup _{t \in \mathbf{R}}\left|2^{-t} \int_{0}^{2^{t}} f(u-\phi(s)) d s\right| .
$$

Then,

$$
\left\|\mathcal{M}_{\phi}(f)\right\|_{p} \leq C_{p}\|f\|_{p}
$$

for $1<p \leq \infty$ and some positive constant $C_{p}$.

Proof. First, assume $\phi$ is a function given as in Theorem 1.1. Without loss of generality, we may assume that $\phi(t)>\phi(0)$ for all $t>0$. For $f \geq 0$ and $u \in \mathbf{R}$, we have

$$
\mathcal{M}_{\phi} f(u)=\sup _{t \in \mathbf{R}}\left(2^{-t} \int_{\phi(0)}^{\phi\left(2^{t}\right)} f(u-s) \frac{d s}{\phi^{\prime}\left(\phi^{-1}(s)\right)}\right) .
$$

Since the function $\frac{1}{2^{t} \phi^{\prime}\left(\phi^{-1}(t)\right)}$ is non-negative, decreasing and its integral over $[\phi(0)$, $\left.\phi\left(2^{t}\right)\right]$ is equal to 1 we have

$$
\mathcal{M}_{\phi} f(u) \leq M_{\mathbf{R}} f(u)
$$

which implies (3.6) by the boundedness of $M_{\mathbf{R}}$ in $L^{p}(\mathbf{R})$ for $1<p \leq \infty$. This completes the proof of the lemma for the case that $\phi$ is given as in Theorem 1.1.

Next, assume $\phi$ is given as in Theorem 1.2. Then, for $f \geq 0$,

$$
\mathcal{M}_{\phi} f(u) \leq 2 \sup _{t \in \mathbf{R}}\left(2^{-t} \int_{2^{t}}^{2^{t+1}} f(u-\phi(s)) d s\right) .
$$

By the conditions on $\phi$, we get

$$
\begin{aligned}
\mathcal{M}_{\phi} f(u) & \leq C \sup _{t \in \mathbf{R}}\left(\int_{\phi\left(2^{t}\right)}^{\phi\left(2^{t+1}\right)} f(u-s) \frac{d s}{s}\right) . \\
& \leq C \sup _{t \in \mathbf{R}}\left(\int_{\phi\left(2^{t}\right)}^{c \phi\left(2^{t+1}\right)} f(u-s) \frac{d s}{s}\right) . \\
& \leq C M_{\mathbf{R}} f(u)
\end{aligned}
$$


which easily implies (3.6)

Finally, to prove (3.6) for $\phi$ given as in Theorem 1.3, we define a family of measures $\lambda_{t}$ on $\mathbf{R}$ by

$$
\hat{\lambda}_{t}(\zeta)=2^{-t} \int_{0}^{2^{t}} e^{-i \zeta \phi(s)} d s
$$

Then by the conditions on $\phi$, it is easy to see that

$$
\left|\hat{\lambda}_{t}(\zeta)-\hat{\lambda}_{t}(0)\right| \leq C\left|2^{t d} \zeta\right| \text { and }\left|\hat{\lambda}_{t}(\zeta)\right| \leq C\left|2^{t d} \zeta\right|^{-1}
$$

By (3.8) and the same argument as in the proof of Theorem 2.3 we get (3.6). The lemma is proved.

THEOREM 3.5. Let $h \in \Delta_{\gamma}\left(\mathbf{R}^{+}\right)$for some $\gamma>1, \phi$ be given as in any one of the Theorems 1.1-1.4 and $\tilde{b}$ be as in Lemma 3.3. Then for $\gamma^{\prime}<p \leq \infty$ and $f \in L^{p}\left(\mathbf{R}^{n+1}\right)$ there exists a positive constant $C_{p}$ which is independent of $\tilde{b}$ such that

$$
\begin{aligned}
& \left\|\sigma_{\tilde{b}, h}^{*}(f)\right\|_{p} \leq C \log \left(|I|^{-1}\right)\|f\|_{p} \text { if }|I|<e^{-1} ; \\
& \left\|\sigma_{\tilde{b}, h}^{*}(f)\right\|_{p} \leq C\|f\|_{p} \quad \text { if }|I| \geq e^{-1} .
\end{aligned}
$$

Proof. We shall only present the proof of (3.9). Without loss of generality we may assume that $\tilde{b} \geq 0$ and $h \geq 0$. By Hölder's inequality we have

$$
\sigma_{\tilde{b}, h}^{*}(f) \leq\|h\|_{\Delta_{\gamma}}\left(\Upsilon_{\tilde{b}}^{*}\left(|f|^{\gamma^{\prime}}\right)\right)^{\frac{1}{\gamma^{\prime}}}
$$

where

$$
\int_{\mathbf{R}^{n+1}} f d \Upsilon_{t, \tilde{b}}=2^{-t} \int_{|u|<2^{t}} f(u, \phi(|u|)) \frac{\tilde{b}(u)}{|u|^{n-1}} d u \quad \text { and } \quad \Upsilon_{\tilde{b}}^{*}(f)=\sup _{t \in \mathbf{R}}|| \Upsilon_{t, \tilde{b}}|* f| .
$$

So we only need to prove that

$$
\left\|\Upsilon_{\tilde{b}}^{*}(f)\right\|_{p} \leq C \log \left(|I|^{-1}\right)\|f\|_{p} \text { for } \gamma^{\prime}<p \leq \infty .
$$

If $\phi$ is given as in any one of the Theorems 1.1-1.3, then the inequality (3.11) follows by Lemma 3.3, Lemma 3.4 and Theorem 2.3.

On the other hand, if $\phi$ is a polynomial, (3.11) follows by a theorem of Stein and Wainger on maximal operators along curves (see [St2], p. 477). The theorem is proved.

4. Proof of theorems. Since $\Omega \in B_{q}^{0,0}\left(\mathbf{S}^{n-1}\right)$, we can write $\Omega=\sum_{\mu=1}^{\infty} c_{\mu} b_{\mu}$, where each $b_{\mu}$ is a $q$-block function, and $M_{q}^{0,0}\left(\left\{c_{\mu}\right\}\right)<\infty$. Without loss of generality, we may assume that $h \in \Delta_{\gamma}$ for some $\gamma, 1<\gamma \leq 2$ and $p$ satisfies $|1 / p-1 / 2|<1 / \gamma^{\prime}$.

To prove our theorems, we shall need to decompose $\Omega$ as follows: For each block function $b_{\mu}$, let

$$
\tilde{b}_{\mu}(x)=b_{\mu}(x)-\int_{\mathbf{S}^{n-1}} b_{\mu}(u) d \sigma(u)
$$


Then one can verify that each $\tilde{b}_{\mu}$ enjoys the following properties:

$$
\begin{aligned}
& \int_{\mathbf{S}^{n-1}} \tilde{b}_{\mu}(u) d \sigma(u)=0 \\
&\left\|\tilde{b}_{\mu}\right\|_{q} \leq 2\left|I_{\mu}\right|^{-\frac{1}{q^{\prime}}} \\
&\left\|\tilde{b}_{\mu}\right\|_{1} \leq 2
\end{aligned}
$$

The new introduced functions $\tilde{b}_{\mu}$ allow us to decompose $\Omega$ into $\Omega=\sum_{\mu=1}^{\infty} c_{\mu} \tilde{b}_{\mu}$ which naturally induces the following decomposition of the corresponding operators:

$$
\mathcal{M}_{\phi, \Omega, h} f\left(x, x_{n+1}\right) \leq \sum_{\mu=1}^{\infty}\left|c_{\mu}\right| \mathcal{M}_{\phi, \tilde{b}_{\mu}, h} f\left(x, x_{n+1}\right) .
$$

By Theorem 3.5 and using a similar argument as in the proof of Theorem 7.5 in [FP] we get

$$
\left\|\left(\int_{\mathbf{R}}\left|\hat{\sigma}_{t, \bar{b}, h} * g_{t}\right|^{2} d t\right)^{\frac{1}{2}}\right\|_{p} \leq C_{p} A_{\mu}\left\|\left(\int_{\mathbf{R}}\left|g_{t}\right|^{2} d t\right)^{\frac{1}{2}}\right\|_{p}
$$

for all $p$ satisfying $|1 / p-1 / 2|<1 / \gamma^{\prime}$ and $f \in L^{p}\left(\mathbf{R}^{n+1}\right)$ where $A_{\mu}=\log \left(\left|I_{\mu}\right|^{-1}\right)$ if $\left|I_{\mu}\right|<e^{-1}$ and $A_{\mu}=1$ if $\left|I_{\mu}\right| \geq e^{-1}$. By (4.6), Lemma 3.3 and Theorem 2.2 we get

$$
\left\|\mathcal{M}_{\phi, \tilde{b}_{\mu}, h} f\right\|_{p}=\left\|\left(\int_{\mathbf{R}}\left|\sigma_{t, \tilde{b}, h} * f\right|^{2} d t\right)^{\frac{1}{2}}\right\|_{p} \leq C_{p} A_{\mu}\|f\|_{p}
$$

for all $p$ satisfying $|1 / p-1 / 2|<1 / \gamma^{\prime}$ and $f \in L^{p}\left(\mathbf{R}^{n+1}\right)$. By (3.1), (4.5) and (4.7) we obtain (1.3) if $\phi$ satisfies the conditions as stated in any one of the Theorems 1.1-1.4. This completes the proof of our theorems.

\section{REFERENCES}

[AqP] H. AL-QASSEM AND Y. PAN, $L^{p}$ estimates for singular integrals with kernels belonging to certain block spaces, Revista Matemática Iberoamericana, 18:3 (2002), pp. 701-730.

[AsAq] A. Al-Salman, AND H. Al-QASSEM, Integral operators of Marcinkiewicz type, to appear in J. of Integr. equations and Applications.

[AsP] A. Al-Salman AND Y. PAN, Singular integrals with rough kernels in $\operatorname{Llog}^{+} L\left(\mathbf{S}^{n-1}\right), \mathrm{J}$. London Math. Soc. 2:66 (2002), pp. 153-174.

[BCP] A. Benedek, A. Calderón And R. Panzone, Convolution operators on Banach space valued functions, Proc. Nat. Acad. Sci. U.S.A., 48 (1962), pp. 356-365.

[CF] L. CHEN AND D. FAN, On singular integrals along surfaces related to block spaces, Integr. equ. oper. theory, 29 (1997), pp. 261-268.

[CFP] J. Chen, D. FAN, AND Y. PAN, A note on a Marcinkiewicz integral operator, Math. Nachr, 227 (2001), pp. 33-42.

[DFP] Y. DING, D. FAN, AND Y. PAN, On the $L^{p}$ boundedness of Marcinkiewicz integrals, to appear in Mich. Math. J.

[DP] Y. DING, AND Y. PAN, $L^{p}$ boundedness of Marcinkiewicz integrals, preprint.

[Du] J. DUOANDIKOETXEA, Multiple singular integrals and maximal functions along hypersurfaces, Ann. Ins. Fourier (Grenoble), 36 (1986), pp. 185-206.

[FP1] D. FAN AND Y. PAN, Singular integral operators with rough kernels supported by subvarieties, Amer. J. Math., 119 (1997), pp. 799-839.

[FP2] D. FAN AND Y. PAN, A singular integral operators with rough kernel, Proc. Amer. Math. Soc., 125 (1997), pp. 3695-3703. 
[KS] M. KeItokU AND E. SATo, Block spaces on the unit sphere in $\mathbf{R}^{n}$, Proc. Amer. Math. Soc., 119 (1993), pp. 453-455.

[KWWZ] W. KIM, S. WAINGer, J. Wright, AND S. Ziesler, Singular integrals and maximal functions associated to surfaces of revolution, Bull. London Math. Soc., 28 (1996), pp. 291-296.

[LPY] S. LU, Y. PAN AND D. YANG, Rough singular integrals associated to surfaces of revolution, to appear in Proc. Amer. Math. Soc.

[LTW] S. LU, M. Taibleson and G. Weiss, Spaces Generated by Blocks, Beijing Normal University Press, 1989, Beijing.

[St1] E. M. SteIn, On the functions of Littlewood-Paley, Lusin and Marcinkiewicz, Trans. Amer. Math. Soc., 88 (1958), pp. 430-466.

[St2] E. M. STEIN, Harmonic analysis real-variable methods, orthogonality and oscillatory integrals, Princeton University Press, Princeton, NJ, 1993. 
